\title{
Chemistry in the Envelopes around Massive Young Stars
}

\author{
Ewine F. van Dishoeck and Floris F.S. van der Tak \\ Leiden Observatory, P.O. Box 9513, 2300 RA Leiden, The Netherlands
}

\begin{abstract}
Recent chemical studies of high-mass star-forming regions at submillimeter and infrared wavelengths reveal large variations in the abundances depending on evolutionary state. Such variations can be explained by freezing out of molecules onto grains in the cold collapse phase, followed by evaporation and high-temperature chemical reactions when the young star heats the envelope. Thus, the chemical composition can be a powerful diagnostic tool. A detailed study of a set of infrared-bright massive young stars reveals systematic increases in the gas/solid ratios and abundances of evaporated molecules with temperature. This 'global heating' plausibly results from the gradual dispersion of the envelopes. We argue that these objects form the earliest phase of massive star formation, before the 'hot core' and ultracompact H II region phase.
\end{abstract}

\section{Introduction}

Massive star-forming regions such as Orion-KL and $\mathrm{SgrB} 2\left(L=10^{4}-10^{6} L_{\odot}\right)$ have traditionally been prime targets for astrochemistry owing to their bright molecular lines (e.g., Blake et al. 1987, Nummelin et al. 1998). Thousands of lines belonging to $\sim 100$ different molecules have been detected. In recent years, submillimeter telescopes combined with new ground- and space-based (ISO) infrared observations have allowed systematic studies of both the gas and the dust in a large sample of massive young stars (see van Dishoeck \& Blake 1998, van Dishoeck \& van der Tak 2000 for reviews). Dramatic variations in the chemical composition are found, even between different objects in the same cloud: some massive young stars (called 'hot cores') show strong submillimeter lines of saturated organic molecules like $\mathrm{CH}_{3} \mathrm{OCH}_{3}$, other objects are rich in $\mathrm{SO}$ and $\mathrm{SO}_{2}$ lines, whereas yet other sources have only simple molecules such as radicals and ions (e.g., Helmich \& van Dishoeck 1997, Hatchell et al. 1998). Striking differences are also seen in the infrared spectra: the coldest, deeply embedded sources show strong absorption by silicates and ices, whereas the more evolved objects have prominent $\mathrm{PAH}$ emission features and strong atomic and ionic emission lines (van den Ancker et al. 2000).

The most successful models for explaining these different chemical characteristics invoke accretion of species onto grains in an icy mantle during the cold (pre-)collapse phase, followed by grain-surface chemistry and evaporation of ices once the young star has started to heat its surroundings. The evaporated molecules subsequently drive a rapid high-temperature 'hot core' gasphase chemistry for a period of $\sim 10^{4}-10^{5} \mathrm{yr}$, resulting in complex, saturated 
Table 1. Chemical characteristics of massive star-forming regions

\begin{tabular}{|c|c|c|c|c|}
\hline Component & $\begin{array}{l}\text { Chemical } \\
\text { characteristics }\end{array}$ & $\begin{array}{l}\begin{array}{l}\text { Submillimeter } \\
\text { diagnostics }\end{array} \\
\end{array}$ & $\begin{array}{l}\text { Infrared } \\
\text { diagnostics }\end{array}$ & Examples \\
\hline Dense cloud & Low- $T$ chemistry & $\begin{array}{l}\text { Ions, long-chains } \\
\left(\mathrm{HC}_{5} \mathrm{~N}, \ldots\right)\end{array}$ & $\begin{array}{l}\text { Simple ices } \\
\left(\mathrm{H}_{2} \mathrm{O}, \mathrm{CO}_{2}\right)\end{array}$ & SgrB2 (NW) \\
\hline Cold envelope & $\begin{array}{l}\text { Low-T chemistry, } \\
\text { Heavy depletions }\end{array}$ & $\begin{array}{l}\text { Simple species } \\
\left(\mathrm{CS}, \mathrm{H}_{2} \mathrm{CO}\right)\end{array}$ & $\begin{array}{l}\text { Ices }\left(\mathrm{H}_{2} \mathrm{O}\right. \\
\left.\mathrm{CO}_{2}, \mathrm{CH}_{3} \mathrm{OH}\right)\end{array}$ & $\begin{array}{l}\text { N7538 IRS9, } \\
\text { W 33A }\end{array}$ \\
\hline $\begin{array}{l}\text { Inner warm } \\
\text { envelope }\end{array}$ & Evaporation & $\begin{array}{l}\text { High } \mathrm{T}_{\text {ex }} \\
\left(\mathrm{CH}_{3} \mathrm{OH}\right)\end{array}$ & $\begin{array}{l}\text { High gas/solid, } \\
\text { High T } \mathrm{Tx}, \\
\text { Heated ices } \\
\left(\mathrm{C}_{2} \mathrm{H}_{2}, \mathrm{H}_{2} \mathrm{O}, \mathrm{CO}_{2}\right)\end{array}$ & $\begin{array}{l}\text { GL } 2591 \\
\text { GL } 2136\end{array}$ \\
\hline Hot core & High-T chemistry & $\begin{array}{l}\text { Complex organics } \\
\left(\mathrm{CH}_{3} \mathrm{OCH}_{3}, \mathrm{CH}_{3} \mathrm{CN},\right. \\
\left.\text { vib. excited } \mathrm{mol}^{\circ}\right)\end{array}$ & $\begin{array}{l}\text { Hydrides } \\
\left(\mathrm{OH}, \mathrm{H}_{2} \mathrm{O}\right)\end{array}$ & $\begin{array}{l}\text { Orion h.c., } \\
\text { SgrB2(N), } \\
\text { G34.3, } \\
W 3\left(\mathrm{H}_{2} \mathrm{O}\right)\end{array}$ \\
\hline $\begin{array}{l}\text { Outflow: } \\
\text { Direct impact }\end{array}$ & $\begin{array}{l}\text { Shock chemistry, } \\
\text { Sputtering }\end{array}$ & $\begin{array}{l}\text { Si- and S-species } \\
\left(\mathrm{SiO}, \mathrm{SO}_{2}\right)\end{array}$ & $\begin{array}{l}\text { Atomic lines, } \\
\text { Hydrides } \\
\left([\mathrm{S} \mathrm{I}], \mathrm{H}_{2} \mathrm{O}\right)\end{array}$ & $\begin{array}{l}\text { W } 3 \text { IRS5, } \\
\text { SgrB2(M) }\end{array}$ \\
\hline $\begin{array}{l}\text { PDR, Compact } \\
\text { H II regions }\end{array}$ & $\begin{array}{l}\text { Photodissociation, } \\
\text { Photoionization }\end{array}$ & $\begin{array}{l}\text { Ions, radicals } \\
\left(\mathrm{CN} / \mathrm{HCN}, \mathrm{CO}^{+}\right)\end{array}$ & $\begin{array}{l}\text { Ionic lines, PAHs } \\
\text { ([NeII], [CII]) }\end{array}$ & $\begin{array}{l}\text { S 140, } \\
\text { W } 3 \text { IRS4 }\end{array}$ \\
\hline
\end{tabular}

organic molecules (e.g., Charnley et al. 1992). The abundance ratios of species such as $\mathrm{CH}_{3} \mathrm{OCH}_{3} / \mathrm{CH}_{3} \mathrm{OH}$ may serve as 'chemical clocks' in this period. Once most of the envelope has cleared, the ultraviolet radiation can escape and forms a photon-dominated region (PDR) at the surrounding cloud material, in which molecules are dissociated into radicals (e.g., $\mathrm{HCN} \rightarrow \mathrm{CN}$ ) and $\mathrm{PAH}$ molecules excited to produce infrared emission. Finally, the (ultra-)compact H II region gives rise to strong ionic lines due to photoionization. Table 1 summarizes the chemical characteristics of the different physical components present in massive YSOs. In single dish submillimeter data, most of these components are blurred together, and sophisticated radiative transfer techniques are required to disentangle them and derive reliable abundances.

\section{A Sample of Embedded, Infrared-Bright Massive Young Stars}

The availability of complete ISO-SWS spectra from $2.5-45 \mu \mathrm{m}$ provides a unique opportunity to study massive young stars through a combination of infrared and submillimeter spectroscopy. Van der Tak et al. (2000a) have selected a set of 9 massive young stars which are bright at mid-infrared wavelengths $(12 \mu \mathrm{m}$ flux $>$ $100 \mathrm{Jy}$ ) and relatively nearby $d \sim 2 \mathrm{kpc}$. The sources are all in an early, deeply embedded evolutionary state (comparable to the 'Class 0 or I' stage of low-mass stars), as indicated by their weak radio continuum emission and absence of ionic lines and PAH features. Complementary JCMT and OVRO data have been obtained. For comparison, 5 infrared-weak sources with similar luminosities are studied at submillimeter only, including hot cores and ultracompact H II regions.

The density structure of the sources has been derived from submillimeter continuum data and from CS lines with a large range of critical density, whereas the temperature structure is calculated self-consistently from the luminosity of the sources. Assuming a power-law density profile $n(r)=n_{o}\left(r / r_{o}\right)^{-\alpha}$, best-fit 
values of $\alpha=1.0-1.5$ are found for the infrared-bright sample, whereas the hot core/compact $\mathrm{H}$ II region sample requires higher values, $\alpha \approx 2$.

The ISO-SWS spectra show absorption by various gas-phase molecules, in addition to strong features by ices. Molecules such as $\mathrm{CO}_{2}, \mathrm{H}_{2} \mathrm{O}, \mathrm{CH}_{4}, \mathrm{HCN}$ and $\mathrm{C}_{2} \mathrm{H}_{2}$ (see van Dishoeck \& van der Tak 2000 for refs.) have been detected. Both the gas-phase and ice results can be plotted as functions of the 'average' temperature of the envelope, measured either from the $45 / 100 \mu \mathrm{m}$ continuum flux ratio or the excitation temperature of $\mathrm{CO}$ and other molecules. The abundances of $\mathrm{H}_{2} \mathrm{O}, \mathrm{HCN}$ and $\mathrm{C}_{2} \mathrm{H}_{2}$ increase by factors of $\gtrsim 10$ with increasing temperature whereas the $\mathrm{H}_{2} \mathrm{O}$ and $\mathrm{CO}_{2}$ ice abundances show a decrease by an order of magnitude, consistent with evaporation of the ices. Moreover, the ices show evidence for systematic heating with the same temperature indicators. These systematic trends are also found in the JCMT submillimeter data of the sources. In particular, a careful analysis of the $\mathrm{CH}_{3} \mathrm{OH}$ data reveals a 'jump' in its abundance from $\sim 10^{-9}$ to $\sim 10^{-7}$ at $T \approx 100 \mathrm{~K}$ in the inner region of the warmer sources, consistent with evaporation of $\mathrm{CH}_{3} \mathrm{OH}$-ices (van der Tak et al. 2000b). These warm sources however lack the typical crowded spectra of 'hot cores' like OrionKL. Either their 'hot cores' are still too small to be picked up by the single dish beams or the hot chemistry has not yet had sufficient time to develop, or both.

A significant result of this study is that even within the narrow evolutionary range of $10^{4}-10^{5} \mathrm{yr}$, clear physical and chemical differentiation of the sources can be found. Since the indicators involve temperatures ranging from $<50 \mathrm{~K}$ (evaporation of ices) to $1000 \mathrm{~K}$ ( $T_{\mathrm{ex}}$ of gas-phase molecules), this suggests that the heating is not a local effect, but that it occurs throughout the envelope. This 'global warming' correlates with the ratio of envelope mass over stellar mass, suggesting that with time, the envelope is dispersed by the star, resulting in a higher temperature throughout the envelope. We therefore argue that the deeply-embedded infrared-bright objects form the earliest evolutionary stage, followed by the 'hot core' stage and finally the ultracompact $\mathrm{H}$ II region stage. Future instrumentation such as ALMA, SOFIA, FIRST and NGST will be essential to further develop these evolutionary diagnostics.

\section{References}

Blake, G.A., Sutton, E.C., Masson, C.R., \& Phillips, T.G. 1987, ApJ, 315, 621 Charnley, S.B., Tielens, A.G.G.M., \& Millar, T.J. 1992, ApJ, 399, L71

Hatchell, J. et al. 1998, A\&AS, 133, 29

Helmich, F.P. \& van Dishoeck, E.F. 1997, A\&AS, 124, 205

Nummelin, A. et al. 1998, ApJS, 117, 427

van den Ancker, M. et al. 2000, A\&A, 358, 1035

van der Tak, F.F.S., van Dishoeck, E.F., Evans, N.J., \& Blake, G.A. 2000a, ApJ, 537,283

van der Tak, F.F.S., van Dishoeck, E.F. \& Caselli, P. 2000b, A\&A, 361, 327

van Dishoeck, E.F. \& Blake, G.A. 1998, ARAA, 36, 317

van Dishoeck, E.F. \& van der Tak, F.F.S. 2000, in 'Astrochemistry: From Molecular Clouds to Planetary Systems', IAU Symposium 197, p. 97 